\title{
Einfluß der Bahnquantisierung im Magnetfeld auf die longitudinale Widerstandsänderung von kovalenten Halbleitern II
}

\author{
Von J. Appel * \\ OSRAM-Studiengesellschaft, Augsburg \\ (Z. Naturforschg. 11 a, 892-901 [1956] ; eingegangen am 3. September 1956)
}

\begin{abstract}
Es wird der Einfluß der Bahnquantisierung auf die Beweglichkeit der Elektronen im longitudinalen Magnetfeld nach der Methode von B Boch-Titeica ${ }^{1}$ für nichtentartete, isotrope Halbleiter als Funktion der Temperatur $T$, der magnetischen Feldstärke $H$ und der scheinbaren Masse $m^{*}$ der Elektronen untersucht.

Mit den explizit berechneten Matrixelementen für die Wechselwirkung der Elektronen mit den akustischen Gitterwellen wird unter Voraussetzung elastischer Stöße $\left(\Delta \varrho / \varrho_{0}\right) n_{\mathrm{e}}$ (die longitudinale Widerstandsänderung bei konstanter Elektronen-Konzentration $n_{\mathrm{e}}$ ) in schwachen Feldern $\left(\gamma=m / m^{*} \cdot \mu H / k T \ll 1[\mu=\right.$ Bohrsches Magneton $\left.]\right)$ sowie in starken Feldern $(\gamma \geqq 3)$ analytisch und bei mittleren Feldstärken $(\gamma=1 ; 2)$ numerisch berechnet.

Es ergibt sich, daß $\left(\Delta \varrho / \varrho_{0}\right) n_{\mathrm{e}}$ in schwachen Feldern eine quadratische und in starken Feldern eine lineare Funktion von $H / T$ ist; es tritt also keine Sättigung in starken Feldern ein. Die quantitativen Ergebnisse werden im Zusammenhang mit den bisherigen Meßergebnissen an kovalenten Halbleitern diskutiert. Im Falle des n-Germaniums $\left(m^{*}=0,2 \mathrm{~m}\right)$ verursacht die Bahnquantisierung bei den maximalen mit Hilfe von Elekromagneten realisierbaren Feldstärken $H \cong 25 \mathrm{kG}$ bei Temperaturen des flüssigen Wasserstoffs Widerstandsänderungen $\left(\Delta \varrho / \varrho_{0}\right) n_{\mathrm{e}} \cong 1 \%$.
\end{abstract}

An Hand des isotropen Modelles - sphärische Energieflächen, isotrope Stoßzeit - konnten die galvanomagnetischen Effekte der kovalenten Elementhalbleiter $\mathrm{Ge}$ und $\mathrm{Si}$ nur qualitativ gedeutet werden ${ }^{2}$. Insbesondere lieferte das isotrope Modell keine Erklärung für die große longitudinale Widerstandsänderung des $\mathrm{n}-\mathrm{Ge}^{3}$. Diese Diskrepanz beseitigten Aвeles und Мегвоом mit dem „manyvalley-model" unter Zugrundelegung einer energieunabhängigen freien Weglänge der Ladungsträger ${ }^{4}$. Die Autoren finden beim Vergleich ihrer Ergebnisse mit den Experimenten von Suhl und Pearson ${ }^{5}$ besonders für das n-Ge bei $300^{\circ}$ und $77^{\circ} \mathrm{K}$ gute quantitative Übereinstimmung.

In neuerer Zeit sind die Messungen über die Widerstandsänderung von $\mathrm{Ge}$ in umfangreichen Untersuchungen von Lautz und Ruppel sowie von Schultz ${ }^{6}$ nach tiefen Temperaturen ausgedehnt worden. Die Autoren finden als wesentliches Ergebnis ihrer Untersuchungen bei Temperaturen des flüs-

* Vorgetragen auf dem Internationalen Kolloquium über Halbleiter und Phosphore 1956 in Garmisch-Partenkirchen; vgl. auch E. N. Adams u. P. N. Argyress, GarmischPartenkirchen 1956.

1 S. Titeica, Ann. Phys., Lpz. 22, 129 [1935].

2 W.S. Whitesell u. V. A. Johnson, Phys. Rev. 89, 941 [1953]; O. Madelung, Z. Naturforschg. 8 a, 791 [1953]; J. Appel, Z. Naturforschg. 9 a, 167 [1954] ; R. K. W IllLardson, T. C. Harmann u. A. C. Beer, Phys. Rev. 96, 1512 [1954]. sigen Wasserstoffs an sehr reinen Einkristallen eine erheblich größere Widerstandsänderung als nach Abeles und Meiвoom zu erwarten ist. Lautz und RUPPEL weisen in einer qualitativen Betrachtung darauf hin, daß die Anisotropietheorie auch bei Berücksichtigung der Streuung der Ladungsträger an den ionisierten Störatomen - dieser Streuprozeß spielt in reinen Ge-Einkristallen bei $20^{\circ} \mathrm{K}$ gegenüber der Wechselwirkung Elektronen-Gitter ohnehin eine untergeordnete Rolle ${ }^{12}$ - die gemessene longitudinale und transversale magnetische Widerstandsänderung bei Temperaturen des flüssigen Wasserstoffs nicht erklären kann. Sie verweisen daher auf die Bahnquantisierung der Elektronen im Magnetfeld als mögliche Ursache der gemessenen Effekte. Bei weiteren Untersuchungen beobachteten die Autoren schwache Oszillationen in der Feldstärkeabhängigkeit von $\Delta \varrho / \varrho_{0}{ }^{7}$, die die Gesetzmäßigkeiten des HaAs-van-Alphen-Effektes zeigten.

3 I. Estermann u. A. Foner, Phys. Rev. 79, 366 [1950].

4 B. Abeles u. S. Меiвоом, Phys. Rev. 95, 31 [1954]; s. auch M. Shibuya, Physica 20, 971 [1954].

5 G. L. Pearson u. H. Suhl, Phys. Rev. 93, 768 [1951].

6 G. Lautz u. W. Ruppel, Z. Naturforschg. 10 a, 521 [1955]; H. Schultz, Internationales Kolloquium über Halbleiter 1956 in Garmisch-Partenkirchen.

7 G. Lautz u. W. Ruppel, Physiker-Tagung Wiesbaden, September 1955; s. auch E. H. W ArRen, Phys. Rev. 98, 1534 [1955]. 
Der Einfluß der Elektronen-Bahnquantisierung wird um so stärker sein, je größer das Verhältnis der Quantisierungs-Energie $\left(\mathrm{m} / \mathrm{m}^{*}\right) \mu H$ zur mittleren thermischen Energie $\overline{E_{0}}$ der Elektronen

$$
\gamma=\frac{\mu^{*} H}{\overline{E_{0}}} ; \quad \mu^{*}=\frac{m}{m^{*}} \mu
$$

ist. In einem nichtentarteten Halbleiter hat $\overline{E_{0}}$ die Größenordnung $k T$ und ist damit - bei nicht zu hohen Temperaturen - klein gegenüber der mittleren thermischen Energie der Leitungselektronen in einem einwertigen Metall: $\overline{E_{0}}=E_{\mathrm{F}} \quad\left(E_{\mathrm{F}}=\right.$ mittlere Fermi-Energie). Aus diesem Grunde ist zu erwarten, daß unter gewissen Voraussetzungen (wie z. B. $m^{*} / m \ll 1$ ) in Halbleitern die Bahnquantisierung der Elektronen einen bedeutend stärkeren Einfluß auf die galvanomagnetischen Effekte hat als in Metallen. Für Metalle hat Titeica ${ }^{1}$ in einer ausführlichen Arbeit die transversale und longitudinale Widerstandsänderung bei Berücksichtigung der Bahnquantisierung ermittelt.

In Halbleitern ändert sich bei Berücksichtigung der Bahnquantisierung nicht nur die mittlere freie Weglänge der Ladungsträger, sondern auch deren Konzentrationen im Leitungs- bzw. Valenzband ${ }^{8,8 \text { a }}$. Die gesamte longitudinale Widerstandsänderung ist für einen Überschußhalbleiter:

$$
\left(\frac{\varrho \mathrm{H}-\varrho_{0}}{\varrho_{0}}\right)_{\text {ges }}=\frac{n_{\mathrm{e}}^{0}-n_{\mathrm{e}}}{n_{\mathrm{e}}}\left\{1+\left(\frac{\Delta \varrho}{\varrho_{0}}\right)_{\mathrm{n}_{\mathrm{e}}}\right\}+\left(\frac{\Delta \varrho}{\varrho_{0}}\right)_{\mathrm{n}_{\mathrm{e}}} ;
$$

$n_{\mathrm{e}}{ }^{0}(T)$ ist die thermische Gleichgewichts-Konzentration der Elektronen ohne Feld. $n_{\mathrm{e}}(H, T)$ haben wir für ein einfaches Halbleiter-Modell in (I) berechnet. In dieser Arbeit wird bei Berücksichtigung der Annahmen von Titeica über die Wechselwirkung Elektronen-Gitter unter genauer Berechnung der entsprechenden Übergangswahrscheinlichkeit für einen Stoßprozeß $\left(\Delta \varrho / \varrho_{0}\right)_{n_{\mathrm{e}}}$ untersucht. Die allgemeinen Voraussetzungen sind: Maxwell-BoltzmanNStatistik für die Elektronen im Leitungsband, sphärische Energieflächen und Stoßzeitisotropie. Das Magnetfeld wird über die Verteilungsfunktion der

8 J. Appel, Z. Naturforschg. 11 a, 689 [1956], im folgenden als (I) zitiert.

8a A nm. b. d. Korr.: Nach freundlicher privater Mitteilung haben Y. Yafet, R.W. Keyes und E. N. Adams den Einfluß eines Magnetfeldes auf die Соulomв-Bindungsenergie von Störstellen-Elektronen an Hand des Wasserstoffatom-Modelles untersucht. Die sich ergebene Zunahme der Ionisierungsenergie mit wachsender magnetischer
Elektronen schon in nullter Näherung streng berücksichtigt. Als Störungen wirken die thermischen Gitterwellen und das elektrische Feld.

\section{A. Berechnung des Widerstandes$$
\varrho(H, T)
$$

Zur Berechnung von $\varrho$ in Abhängigkeit von der Temperatur $T$ und der magnetischen Feldstärke $H$ ist, wie stets, die Kenntnis der stationären Verteilungsfunktion im gestörten Zustand $f_{1}=f+g$ wesentlich. $f_{1}$ wird nach der Methode von Bloch-Titeica aus der statistischen Fundamentalgleichung

$$
\left(\frac{\partial f_{1}}{\partial t}\right)_{\text {Stoss }}+\left(\frac{\partial f_{1}}{\partial t}\right)_{\text {Feld }}=0
$$

ermittelt. $\left(\partial f_{1} / \partial t\right)_{\text {Feld }}$ kann man bei Kenntnis der ungestörten Verteilungsfunktion $f$ in der üblichen Weise berechnen, weil das Magnetfeld nur die Elektronenbewegung senkrecht zu beiden Feldrichtungen quantisiert. Zur Berechnung von $\left(\partial f_{1} / \partial t\right)_{\text {Stoss }}$ brauchen wir die Matrixelemente der Übergangswahrscheinlichkeiten. Diese führen im feldfreien Fall $H=0$ bekanntlich auf die Blochsche Konstante $C$. Hier hängen sie in komplizierter Weise von Anfangs- und Endzustand des Elektrons sowie von der magnetischen Feldstärke $H$ und den Komponenten des Ausbreitungsvektors $\mathfrak{q}\left(\mathfrak{q}_{x}, \mathfrak{q}_{y}, \mathfrak{q}_{z}\right)$ der am Stoßprozeß beteiligten Gitterwelle ab.

\section{Die ungestörte Verteilungsfunktion $f$,} die Änderung von $f_{1}$ durch das elektrische Feld

Das elektrische Feld $E$ und das magnetische Feld $H$ seien in $x$-Richtung orientiert. Dann sind die teildiskreten Energie-Eigenwerte der Elektronen im L-Band:

$$
\begin{gathered}
E=2 \mu^{*} H\left(n+\frac{1}{2}\right)+\frac{p_{x}^{2}}{2 m^{*}} ; \\
p\left(p_{x}, p_{y}, p_{z}\right) \text { Elektronenimpuls. }
\end{gathered}
$$

Die zu (3) gehörigen Eigenfunktionen sind

$$
\psi=\varphi_{n}\left(y-y_{0}\right) e^{i / \hbar \cdot\left(p_{x} x+p_{z} z\right)} .
$$

Feldstärke wirkt in demselben Sinne, wenn auch nicht so stark, wie die Bahnquantisierung der Elektronen im Leitungsband: Die Konzentration der „freien“ Elektronen nimmt mit zunehmender magnetischer Feldstärke ab. Die entsprechende Änderung der Hall-Konstanten haben R.W. Keyes und R. J. Sladek (Phys. Rev. 100, 1262 [1955]) an einer reinen n-leitenden InSb-Probe mit einer Elektronenkonzentration von $10^{14} \mathrm{~cm}^{-3}$ bei $4,2^{\circ} \mathrm{K}$ nachgewiesen. 
$\varphi_{n}$ ist die $n$-te Eigenfunktion, $y_{0}=c p_{z} / e H$ die Ruhelage des harmonischen Oszillators.

Die Anzahl $N(n)$ der entarteten Eigenwerte (3) im Impulsintervall $p_{x}$ bis $p_{x}+\Delta p_{x}$ ist:

$$
N(n)=L^{3} \frac{e H}{h^{2} c} \Delta p_{x} .
$$

$L$ ist das Periodizitätsintervall für die Eigenfunktionen (4). Im Hinblick auf die Berechnung der Matrixelemente der Übergangswahrscheinlichkeiten sei $L$ so gewählt, daß die Oszillatoreigenfunktionen $\varphi_{n}\left(y-y_{0}\right)$ an den Grenzen des Periodizitätsintervalles nahezu verschwinden. Dann ist die periodische Fortsetzung der Eigenfunktionen $\psi$ auch in $y$-Richtung erlaubt ${ }^{1}$. Die Anzahl der Elektronen mit einer Oszillatorquantenzahl $n$ und einer Impulskomponente zwischen $p_{x}$ und $p_{x}+\Delta p_{x}$ ist pro $\mathrm{cm}^{3}$

$$
\begin{aligned}
f \frac{e H}{h^{2} c} \Delta p_{x}= & 8 n_{\mathrm{e}}\left(\frac{\pi \hbar^{2}}{2 m^{*} k T}\right)^{3 / 2} \\
& \cdot \frac{\operatorname{Sin} \gamma}{\gamma} e^{-2 \gamma\left(n+\frac{1}{2}\right)-\frac{p_{x}^{2}}{2 m^{*} k T}} \frac{e H}{h^{2} c} \Delta p_{x} .
\end{aligned}
$$

Eine gewisse Vorstellung über die Verteilung der Elektronen auf die Quantenzustände bekommt man mit (5), wenn man den prozentualen Anteil $x$ derjenigen Elektronen kennt, die bei gegebener Feldstärke $H$ und gegebener Temperatur $T$ die Quantenzustände: $0 \leqq n \leqq \bar{n}(x)$ besetzen. Es ist

$$
\bar{n}(x)=\frac{-\ln (1-x)}{2 \gamma}-1 ; \quad 0 \leqq x \leqq 1 .
$$

So sind z. B. mehr als $99,9 \%$ aller Elektronen in den Zuständen: $0 \leqq n \leqq(4 / \gamma-1)$.

Der Einfluß des elektrischen Feldes auf $f_{1}$ wird wie im feldfreien Fall $H=0$ berechnet, weil die Bewegung eines Wellenpaketes in Richtung des elektrischen Feldes nicht quantisiert wird. Es ist

$$
\left(\frac{\partial f_{1}}{\partial t}\right)_{\text {Feld }}=\left(\frac{\partial f}{\partial t}\right)_{\text {Feld }}=-\frac{e F}{k T} \frac{\hbar l}{m^{*}} f ; \quad l=\frac{p_{x}}{\hbar} .
$$

\section{Wechselwirkung der Elektronen mit dem Kristallgitter}

a) Die Übergangswahrscheinlichkeiten

Nach Titeica ist das Wechselwirkungspotential $U(\mathrm{r})$ bei alleiniger Wechselwirkung der Elektronen

9 A. Sommerfeld u. H. Bethe, Handbuch der Physik XXIV/2, Berlin 1933. mit den longitudinalen akustischen Wellen proportional zur kubischen Dilatation:

$$
U(\mathfrak{r})=D \operatorname{div} \mathfrak{u}(\mathfrak{r}) .
$$

$D$ ist eine Proportionalitätskonstante, $\mathfrak{t}$ ist der in üblicher Weise ${ }^{9}$ definierte Verschiebungsvektor für einen Gitterpunkt, der im Falle ruhender Atome am Ort $\mathfrak{r}$ liegt und durch die thermischen Gitterschwingungen nach $\mathfrak{r}+\mathfrak{U}$ transportiert wird. $U(\mathrm{r})$ ist im Prinzip dasselbe Wechselwirkungspotential, wie es in neuerer Zeit von Bardeen und Shockley ${ }^{10}$ zur Behandlung der Wechselwirkung Elektronen Gitter in nicht polaren Halbleitern benutzt worden ist. Bei Bardeen und Shockley ist $D$ proportional zu $\mathrm{d} E_{\mathrm{g}} / \mathrm{d} V \quad\left(E_{\mathrm{g}}=\right.$ Breite der verbotenen Zone $)$ und den elastischen Konstanten des Kristalles. Der Ansatz (8) ermöglicht eine einfache Berechnung der Übergangswahrscheinlichkeiten $\ddot{U}$. Es ist die Wahrscheinlichkeit für den Übergang eines Elektrons

aus dem Anfangszustand: $n, l=p_{x} / \hbar, r=p_{z} / \hbar$

in den Endzustand: $n^{\prime}, l^{\prime}, r^{\prime}$

pro Zeiteinheit für einen Absorptionsprozeß:

$$
\ddot{U}_{\text {Abs. }}=\frac{\partial}{\partial t}\left\{A q\left|\left(e^{i(\mathfrak{q} \mathrm{r})}\right)\right|^{2} \Omega\left(\frac{E^{\prime}-E}{\hbar}-\omega\right) N\right\}
$$

und für einen Emissionsprozeß:

$$
\begin{aligned}
\dot{U}_{\text {Emiss. }}=\frac{\partial}{\partial t}\{A & q\left|\left(e^{-i(\mathfrak{q} \mathfrak{r})}\right)\right|^{2} \\
& \left.\cdot \Omega\left(\frac{E^{\prime}-E}{\hbar}+\omega\right)(N+1)\right\} .
\end{aligned}
$$

Dabei ist

$$
N=\left(e^{\hbar u_{0} q / k T}-1\right)^{-1}
$$

die Plancksche Verteilungsfunktion für die Gitterquanten,

$$
A=\frac{D^{2}}{M \hbar u_{0}} ; \quad \Omega(x)=\frac{1-\cos x t}{x^{2}},
$$

$u_{0}$ die Schallgeschwindigkeit $\left(\omega=u_{0} q\right), M$ die Masse eines Würfels mit einer Kantenlänge $L$.

Die Matrixelemente

$$
\left(e^{ \pm i(\mathfrak{q} \mathfrak{r})}\right)=\int \psi^{\prime *} e^{ \pm i(\mathfrak{q} \mathfrak{r})} \psi \mathrm{d} \tau
$$

sind nur dann von 0 verschieden, wenn die Auswahlregeln:

$$
l^{\prime}=l+q_{x} \text { und } r^{\prime}=r+q_{z}
$$

für einen Absorptionsprozeß

10 J. Bardeen u. W. Shockley, Phys. Rev. 80, 72 [1950]. (Deformation Potential Theory.) 
oder

$$
l^{\prime}=l-q_{x} \text { und } r^{\prime}=r-q_{z}
$$

für einen Emissionsprozeß

erfüllt sind. Dann ist:

$$
\left(e^{ \pm i(\mathfrak{q} \mathfrak{r})}\right)=\int_{-\infty}^{+\infty} e^{ \pm i q_{y} y} \varphi_{n}\left(y-y_{0}\right) \varphi_{n^{\prime}}\left(y-y_{0}{ }^{\prime}\right) \mathrm{d} y .
$$

Der absolute Betrag der beiden Integrale ist gleich und wird mit $J_{n n^{\prime}}$ bezeichnet. Für die $J_{n n^{\prime}}$ findet Titeica die Differentialgleichung

$$
\begin{aligned}
\frac{\mathrm{d}^{2} J_{n n^{\prime}}}{\mathrm{d} \alpha^{2}}+ & +\frac{1}{\alpha} \frac{\mathrm{d} J_{n n^{\prime}}}{\mathrm{d} \alpha} \\
+\left[n+n^{\prime}+1-\frac{\left(n-n^{\prime}\right)^{2}}{\alpha^{2}}-\frac{\alpha^{2}}{4}\right] J_{n n^{\prime}}=0 & \text { (11) } \\
& \text { mit } \alpha^{2}=\frac{1}{2 \mu^{*} H} \frac{\hbar^{2}}{m^{*}}\left(q_{y}{ }^{2}+q_{z}{ }^{2}\right) .
\end{aligned}
$$

Man braucht zur möglichst genauen Berechnung des Widerstandes bei mittleren Feldstärken $(\gamma \sim 1)$ die $J_{n n^{\prime}}$ für beliebige Parameterwerte $\alpha$. Daher suchen wir die allgemeingültige Lösung der obigen Differentialgleichung. Mit dem Ansatz

$$
J_{n n^{\prime}}(\alpha)=\frac{1}{\alpha} P_{\varkappa, \frac{\lambda}{2}}\left(\frac{\alpha^{2}}{2}\right)
$$

geht (11) in die Whittakersche Differentialgleichung für die Whittakersche Funktion $P_{\varkappa, \frac{\lambda}{2}}\left(\frac{\alpha^{2}}{2}\right)$ über:

$$
\begin{gathered}
\frac{\mathrm{d}^{2} P_{\varkappa, \frac{\lambda}{2}}}{\mathrm{~d}\left(\alpha^{2} / 2\right)^{2}}+\left(-\frac{1}{4}+\frac{\varkappa}{\alpha^{2}}+\frac{1-\lambda^{2}}{\alpha^{4}}\right) P_{\varkappa, \frac{\lambda}{2}}=0, \\
\varkappa=n+n^{\prime}+1, \quad \lambda=n-n^{\prime} .
\end{gathered}
$$

Über die Lösungen dieser Differentialgleichung finden wir die uns interessierenden Partikularintegrale von (9) :

$$
\begin{aligned}
J_{n n^{\prime}}= & \frac{C}{a} \frac{1}{\Gamma\left(1+n-n^{\prime}\right)}\left(\frac{a^{2}}{2}\right)^{\frac{1+n-n^{\prime}}{2}} e^{-\frac{a^{2}}{2}} \\
& \cdot{ }_{1} F_{1}\left(-n^{\prime}, 1+n-n^{\prime}, \frac{\alpha^{2}}{2}\right) ; n \geqq n^{\prime},
\end{aligned}
$$

$$
\begin{aligned}
J_{n n^{\prime}}= & \frac{C}{\alpha} \frac{1}{\Gamma\left(1+n^{\prime}-n\right)}\left(\frac{a^{2}}{2}\right)^{\frac{1+n^{\prime}-n}{2}} e^{-\frac{a^{2}}{2}} \\
& \cdot{ }_{1} F_{1}\left(-n, 1+n^{\prime}-n, \frac{a^{2}}{2}\right) ; \quad n^{\prime} \geqq n .
\end{aligned}
$$

Substituiert man die konfluente hypergeometrische Funktion ${ }_{1} F_{1}(-m, \lambda, z)$ durch das entsprechende Laguerre-Polynom:

$$
L_{m}^{(\lambda)}(z)=\frac{\Gamma(m+\lambda+1)}{m ! \Gamma(\lambda+1)}{ }_{1} F_{1}(-m, 1+\lambda, z),
$$

so erhält man unter Berücksichtigung der Normierungsbedingung:

$$
\int_{0}^{\infty} W_{n n^{\prime}}\left(\frac{\alpha^{2}}{2}\right) \alpha \mathrm{d} \alpha=1
$$

für die $W_{n n^{\prime}}=J_{n n^{\prime}}^{2}$ :

$$
\begin{aligned}
W_{n n^{\prime}}\left(n \geqq n^{\prime}\right)=W_{n^{\prime}}^{n}= & \frac{n^{\prime} !}{n !}\left(\frac{\alpha^{2}}{2}\right)^{n-n^{\prime}} \\
& \cdot e^{-\frac{a^{2}}{2}}\left[L_{n^{\prime}}^{n-n^{\prime}}\left(\frac{\alpha^{2}}{2}\right)\right]^{2}, \\
W_{n n^{\prime}}\left(n^{\prime} \geqq n\right)=W_{n}^{n^{\prime}}= & \frac{n !}{n^{\prime} !}\left(\frac{\alpha^{2}}{2}\right)^{n^{\prime}-n} \\
& \cdot e^{-\frac{a^{2}}{2}}\left[L_{n}^{n^{\prime}-n}\left(\frac{\alpha^{2}}{2}\right)\right]^{2} .
\end{aligned}
$$

Durch die Normierungsbedingung wird der Faktor $C$ festgelegt, der in die Lösung der homogenen Differentialgleichung (11) eingeht. Mit (16a,b) kennen wir nach (9) die Übergangswahrscheinlichkeiten und können damit $\left(\partial f_{1} / \partial t\right)_{\text {Stoss }}$ berechnen. Die mikroskopische Reversibilität der Prozesse ist nach (16 a, b) streng gewährleistet.

b) Die Änderungder Verteilungsfunktion $f_{1}$ durch Stöße, die BLochsche In tegralglei chung

$\left(\partial f_{1} / \partial t\right)_{\text {Stoss }}$ ist mit (9) und (16) unter erlaubter Vernachlässigung des Pauli-Prinzips

$$
\begin{aligned}
\left(\frac{\partial f_{1}}{\partial t}\right)_{\mathrm{Stoss}}=A \frac{\partial}{\partial t}\left\{\sum _ { n ^ { \prime } = 0 } ^ { n } \sum _ { l } \sum _ { q } q W _ { n ^ { \prime } } ^ { n } \left[\Omega\left(\frac{E^{\prime}-E}{\hbar}-\omega\right)\left(g^{\prime}(N+1)-g N\right)\right.\right. & \left.\left.+\Omega\left(\frac{E^{\prime}-E}{\hbar}+\omega\right)\left(g^{\prime} N-g(N+1)\right)\right]+\sum_{n^{\prime}=n+1}^{\infty} \ldots\right\} .
\end{aligned}
$$

Dabei ist berücksichtigt, $\mathrm{da} \beta(\partial f / \partial t)_{\mathrm{Stoss}}=0$ ist. Der Summand unter der zweiten Summe $\sum_{n+1}^{\infty}$ unterscheidet sich vom ersten nur durch $W_{n}^{n^{\prime}}$ an Stelle von $W_{n^{\prime}}^{n}$. Die vorgenommene Aufspaltung der rechten Seite von (17) ist im Hinblick auf die Definition der $W_{n n^{\prime}}$ nach (16) notwendig, weil die folgenden Möglichkeiten für die Änderung der $(n, l)$ bei einem Stoßprozeß bestehen: 
$n^{\prime} \geqq n, \quad l^{\prime} \geqq l: \quad$ Absorptionsproze $\beta$,

$\left.n^{\prime} \geqq n, \quad l^{\prime} \leqq l:\right\} \quad$ Abs.- oder Emiss.-Prozeß,

$n^{\prime} \leqq n, \quad l^{\prime} \geqq l:$

$n^{\prime} \leqq n, \quad l^{\prime} \leqq l: \quad$ Emissionsprozeß.

Auf der rechten Seite von (17) wird zunächst die Summation über $\mathfrak{q}$ durch eine Integration über die
Komponenten in Zylinderkoordinaten:

$$
q_{z}=q_{r} \cos \varphi, \quad q_{y}=q_{r} \sin \varphi, \quad q_{x}
$$

ersetzt, über $\varphi$ integriert und dann, wie bei TiteicA (l. c. ${ }^{1}$, S. 142 u. 155) im Prinzip ausgeführt ist, die $\Omega$-Funktion durch Integration über $q_{r}$ eliminiert. Führt man noch gemäß der Auswahlregel (10) $l^{\prime}$ an Stelle von $q_{x}$ als Integrationsvariable ein, so ist

$$
\begin{aligned}
\left(\frac{\partial f_{1}}{\partial t}\right)_{\text {Stoss }}=\frac{D^{2}}{4 \pi \delta \hbar u_{0}{ }^{2}}\left\{\sum_{n^{\prime}=0}^{n} \int\right. & \mathrm{d} l^{\prime}\left(\left[W_{n^{\prime}}^{n} q^{2}\left(g^{\prime}(N+1)-g N\right)\right]_{\mathrm{Abs} .}\right. \\
& \left.\left.+\left[W_{n^{\prime}}^{n} q^{2}\left(g N-g^{\prime}(N+1)\right)\right]_{\text {Emiss. }}\right)+\sum_{n^{\prime}=n+1}^{\infty} \ldots\right\}=\frac{e F}{k T} \frac{\hbar l}{m^{*}} f .
\end{aligned}
$$

In [ ] $]_{\text {Abs. }}$ und [ ] Emiss. sind für $q_{x}$ und $q_{r}$ die Lösungen von (10) und des Energiesatzes einzusetzen. Gl. (18) ist die modifizierte Blochsche Integralgleichung für die Störfunktion $g$. Zur Berechnung von $g$ machen wir einige in der Leitfähigkeitstheorie der kovalenten Halbleiter gebräuchliche Annahmen. Bei nichtentarteten Halbleitern können die Elektronen im feldfreien Fall unter Erhaltung des Energiesatzes nur mit den langen Gitterwellen des Debyeschen Spektrums wechselwirken ${ }^{11}$, deren Gitterquanten nach der klassischen Statistik verteilt sind:

$$
N=\frac{k T}{\hbar u_{0} q} .
$$

Die Näherung (19) wird im feldfreien Fall für elastische Stöße durch Impuls- und Energiesatz gerechtfertigt. Auch im Magnetfeld wird (19) gelten, sofern die mittlere Energie $\bar{E}$ der Elektronen im Magnetfeld:

$$
\bar{E}=\frac{k T}{2}(1+2 \gamma \operatorname{Ctg} \gamma)
$$

in der Größenordnung der mittleren thermischen Energie der Elektronen ohne Feld: $\bar{E}_{0}=\frac{3}{2} k T$ bleibt. Es ist aber selbst in starken Feldern: $\gamma \sim 3$ die mittlere Elektronen-Energie $\bar{E} \lesssim 3 \bar{E}_{0}$. Aus diesem Grunde wird auch die Energie-Änderung eines Elektrons beim Stoßprozeß - wie im feldfreien Fall - vernachlässigt:

$$
E^{\prime}=E\left(n^{\prime}, l^{\prime}\right) \sim E(n, l) .
$$

Die beiden Näherungen (19) und (21 a) führen im feldfreien Fall auf das $T^{-1}$-Gesetz für eine entsprechend definierte mittlere freie Weglänge der Elek-

11 H. FRöhlich, Elektronentheorie der Metalle. tronen. Das zugehörige $T^{-3 / 2}$-Gesetz für die Elektronenbeweglichkeit ist von Debye und Conwell ${ }^{12}$ an reinen Germanium-Einkristallen bis herunter zu $10^{\circ} \mathrm{K}$ näherungsweise experimentell bestätigt worden.

Zur Lösung der Integralgleichung (18) wird der übliche Ansatz gemacht:

$$
g(E)=f(E) \frac{c(E)}{k T} l .
$$

Einsetzen von (21 b) in (18) erfordert zur Bestimmung von $g\{E(n, l)\}$ die Summation über $n^{\prime}$ und die Integration über $l^{\prime}$. Da der Aufwand für Summation und Integration zu groß ist, wenden wir die Lösungsmethode von Titeica an und setzen $c(E)$ = const. In grober Weise können wir diesen Ansatz dadurch rechtfertigen, daß einerseits die Blochsche Gleichung für Metall-Elektronen bei hohen (und auch bei tiefen) Temperaturen mit $c(E)=$ const gelöst wird und andererseits wegen (19) die Wechselwirkung Elektronen - Gitter in einem Halbleiter oberhalb einiger ${ }^{\circ} \mathrm{K}$ derjenigen von Metallen für $T / \Theta>1 \quad(\Theta=$ Debye-Temperatur $)$ entspricht. Mit

$$
f_{1}=f+g=f(l-\bar{l})=f\left(1+\frac{\hbar^{2}}{m^{*} k T} l \bar{l}\right)
$$

wird aus (18) direkt die mittlere Impulsquantenzahl $\bar{l}$ für die Bewegung der Leitungselektronen in $x$-Richtung berechnet. Mit dieser mittleren Impulsquantenzahl $\bar{l}$ ist der spezifische Widerstand definiert durch:

$$
\varrho=\frac{m^{*}}{n_{\mathrm{e}} e \hbar|l|} .
$$

12 P. P. Debye u. E. M. Conwell, Phys. Rev. 93, 693 [1954] 
Zur Berechnung von $\bar{l}$ multiplizieren wir Gl. (18) mit $l$, integrieren über $l$ und summieren über $n$ :

$\frac{1}{\bar{l}} \sim \iint \mathrm{d} l \mathrm{~d} l^{\prime}\left(\frac{l^{\prime}+l}{2}+\frac{l^{\prime}-l}{2}\right)\left(l^{\prime}-l\right)$

$\cdot \sum_{n=0}^{\infty}\left\{\sum_{n^{\prime}=0}^{n}\left(\left[q W_{n^{\prime}}^{n} f\right]_{\mathrm{Abs.}}+\left[q W_{n^{\prime}}^{n} f\right]_{\text {Emiss. }}\right)+\sum_{n^{\prime}=n+1}^{\infty} \ldots\right\}$.

Der Summand ist symmetrisch in $n, n^{\prime}$ und $l, l^{\prime}$, so daß Summation und Integration über das Glied mit dem unsymmetrischen Faktor $\left(l^{\prime}+l\right)\left(l^{\prime}-l\right)$ Null ergibt. Wir führen über Energie- und Impulssatz $q_{x}$ und $q_{r}$ an Stelle von $l$ und $l^{\prime}$ als Integrationsvariable ein:

$$
\begin{aligned}
l^{\prime}-l \pm q_{x} & =0 ; & E^{\prime}-E \pm \hbar u_{0} q=0 ; \\
\mathrm{d} l^{\prime} & =\mathrm{d} q_{x} ; \quad & \mathrm{d} l=\frac{u_{0} m^{*}}{\hbar} \frac{q_{r} \mathrm{~d} q_{r}}{q_{x} q} .
\end{aligned}
$$

Die genaue Durchrechnung ergibt mit (23) und (24) für den Widerstand:

$$
\begin{aligned}
& \varrho(H, T)=\frac{B}{8 \pi^{3 / 2}} \frac{m^{*}}{\left(2 m^{*} k T\right)^{3 / 2}} \gamma \frac{\operatorname{Sin} \gamma}{\gamma} \cdot 2 \iint \mathrm{d} q_{x} \mathrm{~d} q_{r} q_{x} q_{r} \\
& \cdot \sum_{n=0}^{\infty}\left\{\sum_{n^{\prime}=0}^{n} W_{n^{\prime}}^{n} e^{-\frac{E\left(n, n^{\prime}\right)}{k T}}+\sum_{n^{\prime}=n+1}^{\infty} W_{n}^{n^{\prime}} e^{-\frac{E\left(n, n^{\prime}\right)}{k T}}\right\}, \quad B=\frac{D^{2}}{\delta u_{0}^{2} n_{\mathrm{e}} e} .
\end{aligned}
$$

Dabei ist aus den Energieeigenwerten $E(n, l)$ im Exponenten von $f \sim f^{\prime}$ die Impulsquantenzahl $l$ mit (21) eliminiert:

$$
\begin{aligned}
E\left(n, n^{\prime}\right)=\mu^{*} H(n & \left.+n^{\prime}+1\right)+\frac{\hbar^{2}}{8 m^{*}} q_{x}{ }^{2} \\
& +\frac{2 m^{*}\left(\mu^{*} H\right)^{2}}{\hbar^{2} q_{x^{2}}}\left(n^{\prime}-n\right)^{2} .
\end{aligned}
$$

B. Die Widerstandsänderung: $\Delta \varrho(H, T) / \varrho_{0}(T)$

Zur expliziten Berechnung von $\varrho$ legen wir zunächst die Integrationsgrenzen in (25) fest. Der Integrationsbereich über $\mathfrak{q}$ ist für $H=0$ eine Kugel mit dem Radius

$$
q_{0}^{2} \leqq 4|\mathfrak{f}|^{2}=\frac{8 m^{*}}{\hbar^{2}} E_{\mathfrak{f}} ; \quad E_{\mathfrak{t}}=\frac{\hbar^{2}}{2 m^{*}} \mathfrak{f}^{2} .
$$

Da wir direkt eine mittlere Impulsquantenzahl $\bar{l}(H, T)$ berechnen, wird in (27) $E_{\ddagger}$ durch $\beta \overline{E_{0}}$ ersetzt, wobei $\beta$ ein Zahlenfaktor von der Größenordnung 1 ist. Dann ist

$$
q_{0}^{2}=\frac{8}{m^{*} \hbar^{2}} \beta \cdot \frac{3}{2} k T .
$$

(27) und (28) folgen im feldfreien Fall streng aus Energie- und Impulssatz. Wir nehmen an, daß auch im Magnetfeld (28) gilt, sofern für die Integration über $q_{r}$ an Stelle von $\bar{E}_{0}$ die mittlere Energie der Elektronen für $H \neq 0: \bar{E}$ eingeführt wird:

$$
q_{0 r}^{2}=\frac{8}{m^{*} \hbar^{2}} \beta \cdot \frac{k T}{2}(1+2 \gamma \operatorname{Ctg} \gamma) .
$$

Diese Annahme erscheint physikalisch plausibel, wenngleich ihre Rechtfertigung einer eingehenden
Untersuchung bedarf. Sie besagt, daß mit wachsender Feldstärke infolge der Zunahme der mittleren Oszillations-Energie der Elektronen in der $y-z$ Ebene - die Eigenfunktionen der Wellenpakete werden immer stärker lokalisiert - die Elektronen mit energiereicheren Gitterquanten wechselwirken können. Der Integrationsbereich über $q$ geht von einer Kugel $(H=0)$ in ein abgeplattetes RotationsEllipsoid um die $q_{x}$-Achse über. Mit der bereits durchgeführten Integration über $\varphi$ (s. o.) ist der Integrationsbereich $G$ auf einen Halbkreis $(H=0)$ bzw. auf eine Halbellipse $(H \neq 0)$ reduziert worden:

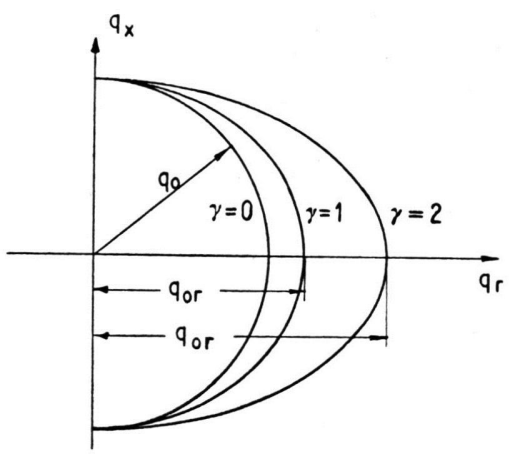

In das Doppelintegral von (25),

$\iint_{G} \ldots \mathrm{d} q_{x} \mathrm{~d} q_{r}=2 \int_{0}^{q_{0}} q_{x} \mathrm{~d} q_{x} \int_{0}^{\sqrt{q_{0 r}^{2}-q_{x}^{2}}} F\left(q_{x}^{2}, q_{r}^{2}\right) q_{r} \mathrm{~d} q_{r}$,

führen wir die neuen Integrationsvariablen

$$
u=q_{x}^{2} \lambda ; \quad v=\frac{a^{2}}{2}=q_{r}{ }^{2} \frac{2 \lambda}{\gamma} ; \quad \lambda=\frac{\hbar^{2}}{8 m^{*} k T}
$$


ein. Dann ist der Widerstand:

$$
\begin{gathered}
\varrho(H, T)=\frac{\sqrt{2}}{2 \pi^{3 / 2}} \frac{B}{\hbar^{4}} m^{* 5 / 2}(k T)^{3 / 2} \gamma^{2} \frac{\operatorname{Sin} \gamma}{\gamma} \int_{0}^{\frac{3}{3} \beta} e^{-u} \mathrm{~d} u \\
\cdot \int_{0}^{\Gamma(\gamma) \sum_{n=0}^{\left(\frac{3}{2} \beta-u\right)}} \sum_{n^{\prime}=0}^{\infty} \sum_{n^{\prime}}^{n} W_{n^{\prime}}^{n}(v) e^{-\left[\gamma\left(n+n^{\prime}+1\right)+\gamma^{2}\left(n-n^{\prime}\right)^{2} / u^{2}\right]} \\
\left.+\sum_{n^{\prime}=n+1}^{\infty} W_{n}^{n^{\prime}}(v) e^{-[\ldots]}\right\} \mathrm{d} v \\
\text { mit } \quad \Gamma(\gamma)=\frac{2}{3 \gamma}(1+2 \gamma \operatorname{Ctg} \gamma) .
\end{gathered}
$$

\section{Der feldfreie Widerstand $\varrho(\dot{H}=0, T)=\varrho_{0}(T)$}

Wir integrieren zunächst die rechte Seite von (29) über $v$. Es ist für beliebige $n, n^{\prime} \operatorname{im} \lim \gamma \rightarrow 0$ :

$$
\lim _{\gamma \rightarrow 0} \int_{0}^{\Gamma(\gamma)\left(\frac{3}{2} \beta-u\right)} W_{n n^{\prime}}(v) \mathrm{d} v=1 .
$$

Die Doppelsumme über $\exp \left[-E\left(n, n^{\prime}\right)\right]$ wird im $\lim \gamma \rightarrow 0$ durch das entsprechende Doppelintegral gut approximiert. Sukzessive Integration über die beiden Variablen $n$ und $n^{\prime}$ liefert:

$$
\begin{aligned}
& \int_{0}^{\infty} \int_{0}^{\infty} e^{-\left[\gamma\left(n+n^{\prime}+1\right)+\gamma^{2} / 4 u \cdot\left(n-n^{\prime}\right)^{2}\right]} \mathrm{d} n \mathrm{~d} n^{\prime} \\
& =\frac{\sqrt{\pi}}{\gamma^{2}} e^{u-\gamma} \sqrt{u}[1-\Phi(\sqrt{u})], \Phi(x)=\frac{2}{\sqrt{\pi}} \int_{0}^{x} e^{--\xi^{2}} \mathrm{~d} \xi .
\end{aligned}
$$

Einsetzen in (29) und Integration über $u$ ergibt den Widerstand bei verschwindendem Magnetfeld:

$$
\begin{aligned}
\varrho_{0}(T) & =\frac{\sqrt{2}}{2 \pi^{3 / 2}} \frac{B}{\hbar^{4}} m^{* 5 / 2}(k T)^{3 / 2} J\left(\frac{3}{2} \beta\right), \\
J(x)=\frac{2 \sqrt{\pi}}{3}\left[x^{3 / 2}(1-\Phi(\sqrt{x}))\right. & \left.\quad+\frac{1}{\sqrt{\pi}}\left(1-e^{-x}(1+x)\right)\right] . \\
& \begin{array}{r|r}
\hline \beta & I\left(\frac{3}{2} \beta\right) \\
\hline 1 & 0,477 \\
2 & 0,571 \\
\hline
\end{array}
\end{aligned}
$$

Wesentlich ist, da $\beta$ nach $(31) \varrho_{0}(T)$ in richtiger Weise sowohl von der Temperatur des Halbleiters als auch von der scheinbaren Masse der Elektronen abhängt. Nach SEITz ${ }^{13}$ gilt für die Beweglichkeit $\mu_{\mathrm{e}}$

13 F. SeItz, Phys. Rev. 73, 549 [1948]. der Elektronen in kovalenten Halbleitern bei reiner Gitterstreuung:

$$
\mu_{\mathrm{e}} \sim m^{*-5 / 2} T^{-3 / 2} .
$$

\section{Die Widerstandsänderung}

a) Schwache Felder: $\gamma \ll 1$

Es darf wie in der Landauschen Theorie des Diamagnetismus freier Elektronen ${ }^{14}$ die Doppelsumme in (29) nach der Summenformel von Euler entwickelt werden. Danach ist in erster Näherung:

$$
\begin{aligned}
\int \ldots \mathrm{d} v=\int & \left\{\int_{-\frac{1}{2}}^{\infty} \int_{-\frac{1}{2}}^{\infty} W_{n n^{\prime}} e^{-(E-\gamma) / k T}\right. \\
& +\frac{1}{24}\left(\sum_{0}^{\infty} W_{n 0}\left[\frac{\partial}{\partial n^{\prime}}\left(e^{-E / k T}\right)\right]_{n^{\prime}=0}\right. \\
& +\sum_{0}^{\infty} W_{0 n^{\prime}}\left[\frac{\partial}{\partial n}\left(e^{-E / k T}\right)\right]_{n=0} \\
& \left.+2 \sum_{0}^{\infty}\left(W_{n 1}-W_{n 0}\right) e^{-E\left(n, n^{\prime}=0\right) / k T}\right\} \mathrm{d} v .
\end{aligned}
$$

Über $v$ wird von 0 bis $\infty$ integriert. Diese Vereinfachung hat keinen wesentlichen Einfluß auf das Ergebnis (33). Die Berechnung des Korrekturgliedes in (32) erfolgt in ähnlicher Weise wie bei Titeica. So wird:

$$
\sum_{0}^{\infty} W_{n 0} e^{-E\left(n, n^{\prime}=0\right) / k T}=e^{-E\left(n=\alpha^{2} / 2, n^{\prime}=0\right) / k T}
$$

gesetzt, weil das Maximum $W_{n 0}$ nach Abb. 1 bei $\alpha^{2} \simeq 2 n$ liegt. Berücksichtigt man noch, daß in erster Näherung in $\gamma^{2}$ gilt:

$$
\frac{\operatorname{Sin} \gamma}{\gamma}=1+\frac{\gamma^{2}}{6} \quad \text { für } \quad \gamma \ll 1,
$$

so ist die Widerstandsänderung in schwachen Feldern

$$
\left(\frac{\Delta \varrho}{\varrho_{0}}\right)_{n_{e}} \simeq \frac{\gamma^{2}}{12} \quad \text { für } \quad \gamma \ll 1 .
$$

b) Starke Felder

Nach (6) befinden sich bei $\gamma=3$ mehr als 99\% aller Elektronen im Zustand $n=0$. Vernachlässigt man die $\sim 0,5 \%$ der Elektronen, die sich in den Zuständen $n>0$ aufhalten, so ist mit $W_{00}(v)=e^{-v}$

14 L. Landau, Z. Phys. 64, 629 [1929]. 

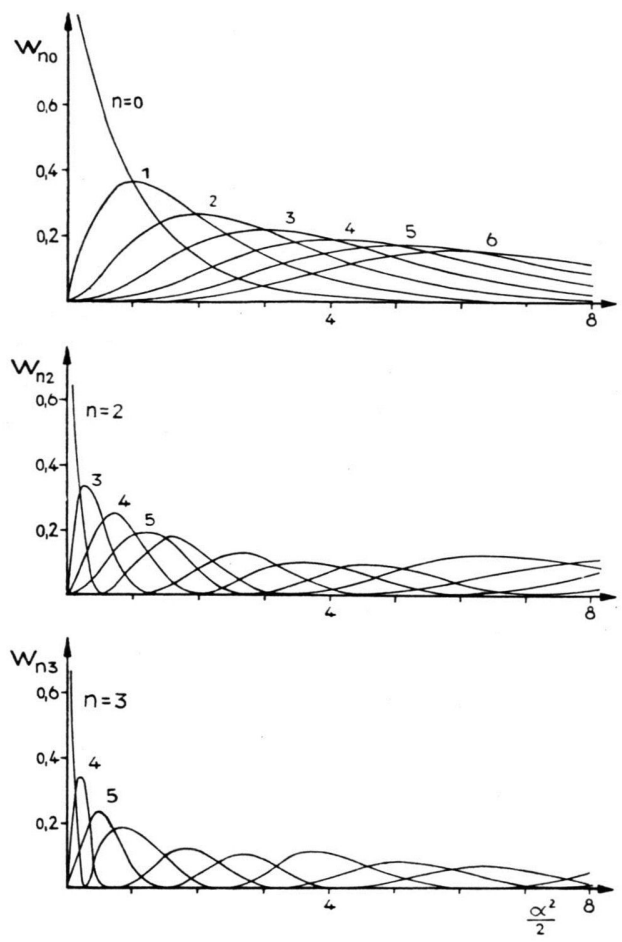

Abb. 1. Die $W_{n n}$, sind als Quadrate der Matrixelemente für die Wechselwirkung Elektronen-Gitter proportional den Übergangswahrscheinlichkeiten. Es ist

$$
a^{2}=\frac{1}{2 \mu^{*} H} \frac{\hbar^{2}}{m^{*}}\left(q_{z}^{2}+q_{y}^{2}\right) .
$$

die Widerstandsänderung:

$$
\left(\frac{\Delta \varrho}{\varrho_{0}}\right)_{n_{e}}=\frac{\gamma \operatorname{Sin} \gamma e^{-\gamma} \int_{0}^{\frac{3}{2} \beta} e^{-u} \mathrm{~d} u \int_{0}^{\Gamma(\gamma)\left(\frac{3}{2} \beta-u\right)} e^{-v} \mathrm{~d} v}{I\left(\frac{3}{2} \beta\right)}-1 ; \gamma \geqq 3 .
$$

Danach gilt für $\gamma \geqq 3$ näherungsweise

$$
\left(\frac{\Delta \varrho}{\varrho_{0}}\right)_{n_{e}} \simeq\left\{\begin{array}{lll}
0,65 \gamma-1 & \text { für } & \beta=1, \\
0,74 \gamma-1 & \text { für } \beta=2 .
\end{array}\right.
$$

c) Mittlere Feldstärken: $\gamma=1 ; 2$

Je kleiner $\gamma$ ist, desto schlechter konvergiert die Summe über $n$ und $n^{\prime}$. Zur Berechnung der Widerstandsänderung für $\gamma=2$ haben wir die Elektronen in den Zuständen $n=0 ; 1$ und 2 , für $\gamma=1$ die in den Zuständen $n=0 ; 1 ; 2$ und 3 berücksichtigt. Die entsprechenden Integrale über $u$ und $v$ lassen sich leicht analytisch $\left(n=n^{\prime}\right)$ oder numerisch $\left(n \neq n^{\prime}\right)$ berechnen.

\section{Diskussion der Ergebnisse}

$\left(\Delta \varrho / \varrho_{0}\right)_{n_{\mathrm{e}}}$ ist in den drei Feldstärkebereichen eine Funktion des Abszissenparameters:

$$
\gamma=6,73 \cdot 10^{-5} \frac{m}{m^{*}} \frac{H}{T} .
$$

Abb. 2. Die berechnete longitudinale Widerstandsänderung $\left(\frac{\Delta \varrho}{\varrho_{0}}\right)_{n_{e}}$ als Funktion von $H / T$. Der Kurvenparameter $\beta$ ist ein Zahlenfaktor, der die oberen Grenzen für die Integration über den $\mathfrak{q}$-Raum festlegt.

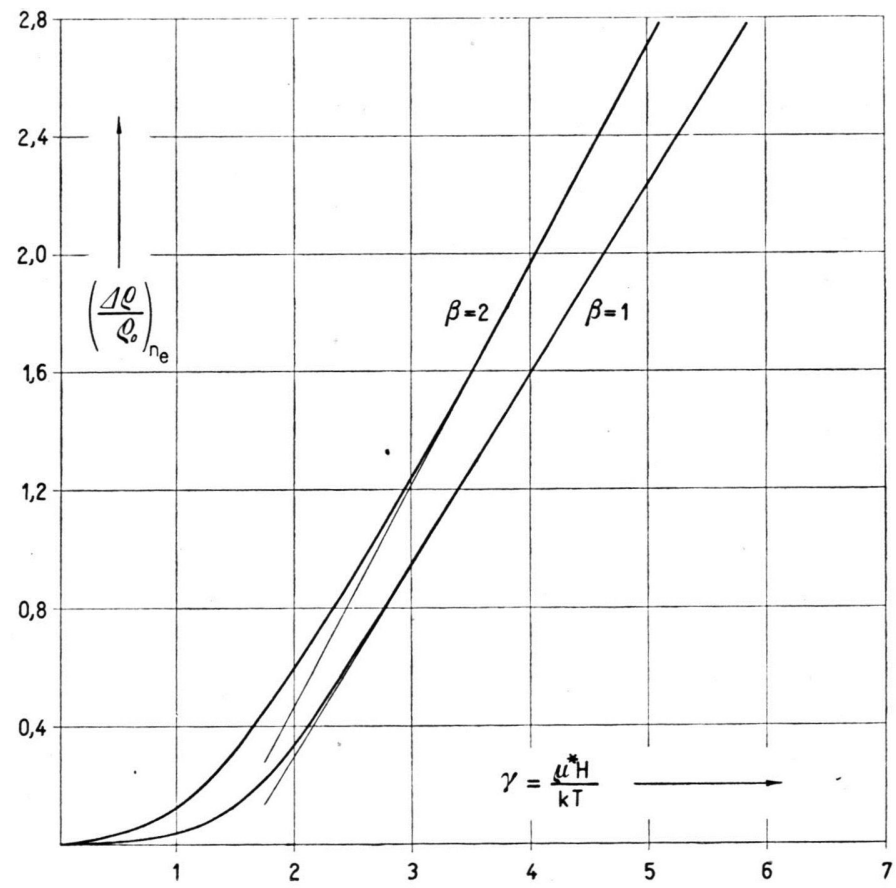




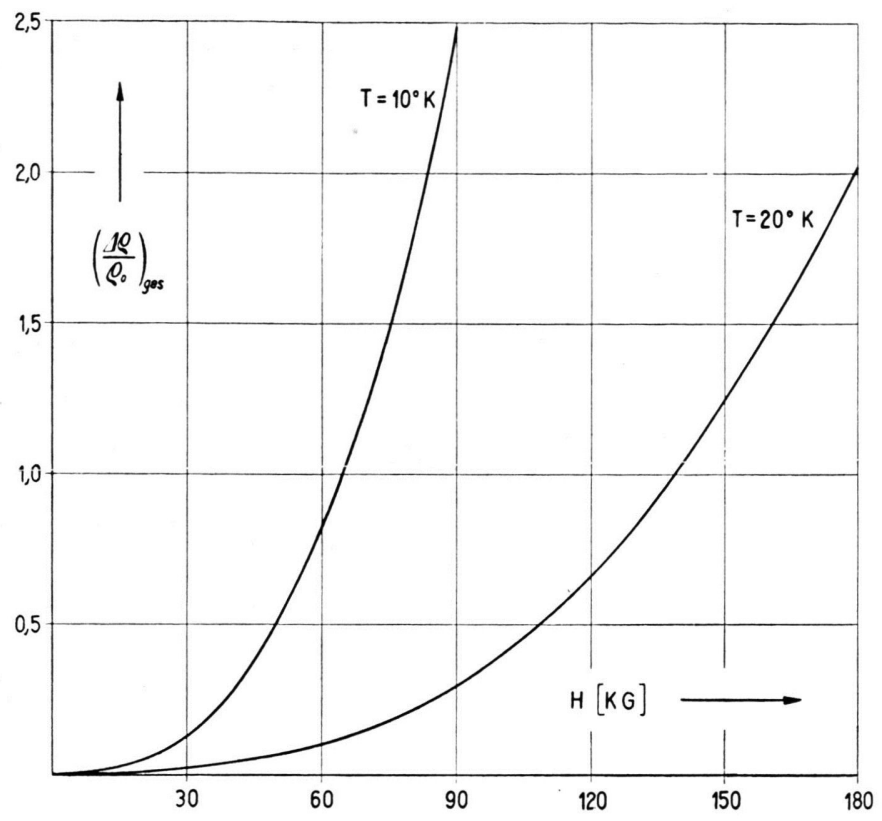

Abb. 3. Die nach Gl. (1) berechnete, gesamte longitudinale Widerstandsänderung für ein einfaches Halbleitermodell mit den Daten: Donatoren-Konzentration $n_{\mathrm{d}}=2 \cdot 10^{14}, \Delta E=0,01 \mathrm{eV}$, $m^{*} / m=0,2$ und $\beta=1$.
Außerdem hängt die Widerstandsänderung von $\beta$ $\mathrm{ab}$, einem Zahlenfaktor, der die oberen Grenzen $q_{0}$ und $q_{0 r}$ für die Integration über das abgeplattete Rotationsellipsoid im $q$-Raum festlegt. Den wahren $\beta$-Wert kennen wir nicht genau, wir wissen, da $\beta$ er bei eins liegt. In Abb. 2 ist die Widerstandsänderung für $\gamma<6$ und $\beta=1 ; 2$ angegeben. In schwachen Feldern ist $\left(\Delta \varrho / \varrho_{0}\right) n_{\mathrm{e}}$ eine quadratische, in starken Feldern eine lineare Funktion von $H / T$. Die Neigung der Geraden in starken Feldern - alle Elektronen sind im Zustand $n=0$ - wird wesentlich durch die scheinbare Masse der Elektronen bestimmt. Bei mittleren Feldstärken $(\gamma \sim 1)$ haben $\left(\Delta \varrho / \varrho_{0}\right) n_{\mathrm{e}}$ und $\Delta n_{\mathrm{e}} / n_{\mathrm{e}}$ (siehe I) Werte von einigen Prozent. Experimentell erreicht man mittlere Feldstärken im Sinne der Bahnquantisierung, d. h. $\gamma$-Werte von der Größenordnung eins, im Falle des $\mathrm{n}$-Germaniums mit Magnetfeldstärken von $25 \mathrm{kG}$ - wie sie maximal mit Hilfe von Elektromagneten erzeugt werden -, bei den Temperaturen des flüssigen und festen Wasserstoffs. Im Bereich der üblichen Laborfeldstärken $(H \lesssim 25 \mathrm{kG})$ ist also im Falle des n-Germaniums im Temperaturbereich zwischen 10 und $20^{\circ} \mathrm{K}$ bei alleiniger Streuung der Elektronen an den thermischen Gitterwellen ein wesentlicher Einfluß der Bahnquantisierung auf die galvanomagnetischen Effekte nicht zu erwarten. Das ersieht man z. B. deutlich aus der Abb. 3, in der die nach (1) berechnete, gesamte longitudinale Wider- standsänderung für einen einfachen Überschuß-Halbleiter, dessen Daten (Donatoren-Konzentration $n_{\mathrm{d}}=$ $2 \cdot 10^{14} \mathrm{~cm}^{-3}, \Delta E=0,01 \mathrm{eV}$ und $\left.m^{*} / m=0,2\right)$ etwa denjenigen von reinem n-Germanium entsprechen, für $T=10 ; 20^{\circ} \mathrm{K}$ über $H / T$ aufgetragen ist. Der Einfluß der Bahnquantisierung spielt bei beiden Temperaturen bis zu $25 \mathrm{kG}$ gegenüber den konventionellen Effekten ${ }^{4}$ keine wesentliche Rolle. Im Sinne der konventionellen Theorie liegen unter diesen Voraussetzungen schon bei einigen $\mathrm{kG}$ starke Felder vor - d. h. die mittlere freie Weglänge der Elektronen ist groß gegenüber dem durch die Lorentz-Kraft verursachten mittleren Krümmungsradius der Elektronenbahnen - während bei den optimalen Werten $10^{\circ} \mathrm{K}$ und $25 \mathrm{kG}$ beim $\mathrm{n}-\mathrm{Ge}$ $\gamma$ nur den Wert 0,84 erreicht. Erst bei höheren Feldstärken, wie sie nach Furth und $\mathrm{W}_{\text {ANICK }}{ }^{15}$ durch kurzzeitige Kondensatorentladungen über spezielle Solenoide erzeugt werden können (bis zu $600 \mathrm{kG}$ ), ist bei $20^{\circ} \mathrm{K}$ ein deutlicher Einfluß der Quantisierung sowohl auf die Widerstandsänderung als auch auf die Hall-Konstante zu erwarten (siehe I).

Im Rahmen der vorliegenden Untersuchung über den Einfluß der Bahnquantisierung auf die longitudinale Widerstandsänderung bei alleiniger Berücksichtigung der Wechselwirkung Elektronen-Gitter

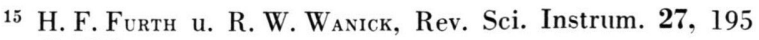
[1956]. 
können die an reinen n-Ge-Einkristallen bei Temperaturen des flüssigen Wasserstoffs gemessenen Effekte ${ }^{6}$ nicht erklärt werden. Ob die Einbeziehung der Störstellenstreuung diese Diskrepanz zu beseitigen vermag, wie es in entsprechender in der konventionellen Theorie bei höheren Temperaturen der Fall ist ${ }^{2,6}$, muß einer genauen Untersuchung vorbehalten bleiben. Für die Deutung der beobachteten Oszillationen in der Feldstärkeabhängigkeit von $\Delta \varrho / \varrho_{0}$ gibt es jedoch bis jetzt keine Anhaltspunkte. In nichtentarteten Halbleitern kann es einen HAASvan-Alphen-Effekt im Sinne der Metalltheorie nicht geben. Nach STEele ${ }^{16}$ (Theory of Numbers) setzt sich die integrale Eigenwertdichte von „freien“ Elektronen bei Berücksichtigung der Bahnquantisierung aus einem in $H$ monotonen und einem in $H$ oszil-

16 M. C. Steele, Phys. Rev. 88, 451 [1952]. latorischen Anteil zusammen. Der $\delta$-Charakter in der Ableitung der Fermischen Verteilungsfunktion erhält diese Oszillationen auch bei der Integration über das gesamte Eigenwertspektrum. Bei der entsprechenden Integration über die Ableitung der Boltzmansschen Verteilungsfunktion werden die Oszillationen „ausgeglättet“, so daß die Konzentration der Elektronen im L-Band eine monoton abnehmende Funktion der magnetischen Feldstärke ist ${ }^{17}$.

Für die stete Förderung dieser Arbeit und für wertvolle Diskussionen danke ich Herrn Prof. Krautz und Herrn Dr. Schultz. Herrn Prof. Kohler bin ich für die Anregung und für einige Diskussionen zu dieser Untersuchung sehr dankbar.

17 S. auch A. N. Wilson, The Theory of Metals, Cambridge 1953, S. $164 \mathrm{ff}$.

\title{
Erweiterung der Theorie magnetohydrodynamischer Wellen und Anwendung auf inhomogene Schichten
}

\author{
Von Egon Richter \\ Aus dem Institut für Theoretische Physik der Technischen Hochschule München \\ (Z. Naturforschg. 11 a, 901-912 [1956]; eingegangen am 15. August 1956)
}

\begin{abstract}
Im Gegensatz zu den bisherigen Untersuchungen über magnetohydrodynamische Wellen werden die Maxwellschen Gleichungen in ihrer strengen Form mit den hydrodynamischen Gleichungen gekoppelt. Die als gyroelektrisches Medium betrachtete Flüssigkeit wird reibungsfrei, unmagnetisierbar und im Ruhesystem elektrisch neutral angenommen, wobei die Materialkonstanten sowie das äußere Magnetfeld ortsabhängig sein können. Nach üblicher Linearisierung lassen sich für Wellenausbreitung längs des äußeren Magnetfeldes die Querwellen gesondert behandeln, die sowohl die elektromagnetischen als auch die Alfvésschen Wellen als Grenzfälle enthalten. Das Reflexions- und Durchlaßvermögen einer inhomogenen Schicht wird berechnet und für homogene Medien der Energiesatz abgeleitet.
\end{abstract}

Im Jahre 1942 zeigte Alfvén ${ }^{1}$, daß sich Störungen in einer leitenden Flüssigkeit, die von einem äußeren Magnetfeld durchsetzt wird, in bestimmter Weise fortpflanzen und daß insbesondere sinusförmige Störungen Wellen erzeugen, die als magnetohydrodynamische Wellen bezeichnet werden. Infolge gewisser Voraussetzungen ergeben sich bei Alfvén allerdings spezielle magnetohydrodynamische Wellen, die nach ihrem Entdecker ALfvénsche Wellen genannt werden. Für die Ableitung dieser Alfvénschen Wellen ist wesentlich, daß der Verschiebungsstrom vernachlässigt,' die Leitfähigkeit unendlich ge-

\footnotetext{
1 H. Alfuén, Ark. Mat. Astr. Fys. B 29, No. 2 [1942]. Cosmical Electrodynamics, Clarendon Press, Oxford 1950.
}

setzt und die Flüssigkeit als inkompressibel betrachtet wird. Die von ALFvén begonnenen Untersuchungen über das Verhalten dieser Wellen wurden vom Standpunkt der Kontinuumstheorie aus von FERRARO $^{2}$ und Roberts ${ }^{3}$ fortgeführt. In diesen Arbeiten wird das Reflexions- und Brechungsgesetz Alfvénscher Wellen für eine Unstetigkeitsfläche der Massendichte abgeleitet und das Reflexionsvermögen bei senkrechtem Einfall angegeben. Naturgemäß gehen in diese Ableitungen die Grenzbedingungen wesentlich ein, die in der Magnetohydrodynamik offenbar auch die Teilchenbewegung mit berücksich-

\footnotetext{
2 V. C. A. Ferraro, Astrophys. J. 119, 393 [1954].

3 P. H. Roberts, Astrophys. J. 121, 720 [1955].
} 